\title{
EPISÓDIOS REACIONAIS NA HANSENÍASE
}

\author{
REACTIONAL EPISODES IN LEPROSY
}

Norma Tiraboschi Foss

Docente. Divisão de Dermatologia. Departamento de Clínica Médica. Faculdade de Medicina de Ribeirão Preto - USP.

Correspondência: Av. Bandeirantes, 3900. CEP 14049-900 Ribeirão Preto - SP - Fone: 16 -602-2446 / Fax: 16 - $633-0236$

Email: ntfoss@fmrp.usp.br

FOSS NT. Episódios reacionais na Hanseníase. Medicina, Ribeirão Preto, 36: 453-459, abr./dez. 2003

RESUMO - Episódios reacionais são quadros agudos, que ocorrem de modo súbito, interrompendo a evolução crônica da hanseníase. Constituem, geralmente, em exacerbada reação inflamatória, localizada ou sistêmica, que pode ser acompanhada de comprometimento de nervos periféricos, resultando em manifestação de dor acentuada, que requer, para sua condução, cuidados especiais. Existem dois tipos de reações hansênicas: Reação tipo1 ou Reação Reversa (RR) e Reação tipo 2 ou Eritema Nodoso Hansênico (ENH). Essas reações foram descritas, segundo suas características clínicas e laboratoriais, sendo destacados os critérios de diagnóstico e as principais diferenças entre RR e ENL, e, ainda, seguidas da apresentação das condutas preconizadas para a condução das alterações clínicas e da opção terapêutica para os dois tipos de reações.

UNITERMOS - Hanseníase. Episódios Reacionais. Reação Reversa. Eritema Nodoso Hansênico.

Hanseníase, causada pelo Mycobacterium leprae, apresenta amplo espectro de manifestações clínicas, envolvendo uma forma inicial-HI (INDETERMINADA), que pode evoluir para as formas polares: VIRCHOWIANA-HV (forma de alta suscetibilidade à infecção) e TUBERCULÓIDE-HT (forma de alta resistência à infecção), além das formas intermediárias. Estas podem se manifestar com características próprias-HD (DIMORFA) ou de uma ou outra forma polar (DIMORFA VIRCHOWIANA-HDV E DIMORFA TUBERCULÓIDE-HDT) $)^{(1)}$.

A doença tem evolução crônica e insidiosa, podendo ser interrompida por episódios agudos, denominados Reações Hansênicas ${ }^{(2)}$. Esses episódios reacionais, caracterizados por reação inflamatória súbita, podem ocorrer em qualquer forma clínica da doença, excluindo a inicial-HI, porém são mais freqüen- tes em pacientes com as formas HV, HDV, HD e HDT. Os quadros reacionais podem ocorrer espontaneamente, antes, durante ou após o tratamento específico ou, ainda, estarem associados a situações clínicas como: vacinação, anemia, puberdade, gestação, parto, intervenção cirúrgica, stress físico e/ou psicológico e infecções intercorrentes (virais, bacterianas, etc), e uso de drogas, sendo as mais comuns: antibióticos, iodeto de potássio, progesterona e vitamina- $\mathrm{A}^{(3)}$. Eventualmente, o quadro reacional pode ser o motivo que leva o paciente a procurar ajuda médica e, só então, ser diagnosticada a doença.

Considerando que os episódios reacionais podem ocorrer durante ou após o tratamento poliquimioterápico, que é constituído pela associação de três drogas com diferentes graus de atividade antimicobacteriana: rifampicina, dapsona e clofazemina ${ }^{(2,4)}$, o apa- 
recimento dessas reações está, também, associado à resposta terapêutica e à capacidade de resposta imunológica do paciente.

Existem dois tipos de reações hansênicas, que refletem dois diferentes tipos de respostas imunológicas e que, provavelmente, constituem as bases para as manifestações clínicas de ambos os estados reacionais ${ }^{(3,5)}$ :

REAÇÃO REVERSA (RR) OU REAÇÃO TIPO 1: envolve, geralmente, as formas dimorfas (HD,HDT e HDL) e pode, eventualmente, ocorrer na HT e, raramente, na HV. O quadro clínico caracteriza-se por sinais de inflamação aguda, tais como dor, eritema, infiltração e edema de lesões pré-existentes, às vezes acompanhadas de novas lesões ${ }^{(6)}$. Geralmente, não há comprometimento sistêmico, as manifestações são localizadas, como neurites isoladas ou acompanhadas de lesões cutâneas. Os casos muito graves podem cursar com ulceração profunda e necrose acentuada, levando a seqüelas irreversíveis. Nos pacientes com HD e HDT, o surto reacional aparece mais freqüentemente durante o sexto mês de poliquimioterapia, enquanto, na HDL, há necessidade de intervalo de tempo de tratamento maior para surgirem as lesões ${ }^{(3)}$. Todo o processo da RR tem sido associado a um súbito aumento da imunidade mediada por células (cell mediated immunity-CMI), constituindo exemplo típico da reação de hipersensibilidade tardia "in vivo", reação tipo $\mathrm{IV}^{(7)}$. Com a involução da reação, as lesões tornam-se descamantes ${ }^{(2,3)}$. Devido ao aspecto clínico do quadro cutâneo, o diagnóstico diferencial deve ser feito, principalmente, com erisipela, vasculites, sífilis, eritema polimorfo e linfoma cutâneo.

Apesar de apresentar um quadro clínico, geralmente, bem localizado, esse tipo de reação é muito preocupante, porque é freqüentemente acompanhado de espessamento de um ou mais nervos periféricos, pode ocorrer de forma aguda, seguido de sintomas de dor e acentuação da sensibilidade em todo o trajeto neural, comprometido e, dependendo da gravidade, pode levar à formação de abscesso neural ${ }^{(3)}$. $\mathrm{O}$ acometimento do nervo pode atingir as fibras sensitivas e motoras dos troncos nervosos, periféricos, gerando lesões definitivas e incapacidade física. Quadros clínicos, como mão em garra, pé caído e paralisia facial, acompanhados de espessamento neural e perda de sensibilidade das áreas afetadas, são as seqüelas da reação aguda, a qual necessita de tratamento adequado o mais rápido possível. Logo, as neuropatias cons- tituem-se em quadros graves, que requerem intervenção e conduta médica imediata, para prevenir o estabelecimento de incapacidades.

Não se observam alterações de exames laboratoriais, como hematológico, funções hepática e renal, sendo que o histopatológico de lesões cutâneas mostra aumento do número de células epitelióides gigantes e linfócitos, e redução do número de bacilos, indicando a atividade da reação na eliminação do bacilo. Raramente, os episódios de reação reversa persistem mais que poucos meses ${ }^{(3)}$.

Reação reversa deve ser diferenciada de recidivas, especialmente nas formas HDT e HT da hanseníase. Pacientes com alta probabilidade de recidivas são aqueles que fizeram o tratamento inadequado, pois a grande maioria das recidivas ocorre com microorganismos sensíveis à poliquimioterapia$\mathrm{PQT}^{(8,9,10)}$. O diagnóstico de recidiva deve ser feito com base nos exames de baciloscopia e histopatológico da lesão, porém, nas formas HDT e HT é frequiente a dificuldade em distinguir a RR de recidiva ${ }^{(3,11)}$. Atualmente, com o seqüenciamento de genes e estudos de genoma do M.leprae, há a possibilidade de diferenciar reação de recidiva. Entretanto, no momento, os casos de recidivas devem seguir os critérios clínicos já estabelecidos ${ }^{(3)}$ e indicados a seguir.

- Início: lento e insidioso, geralmente, vários meses após a suspensão da poliquimioterapia.

- Localização: novas lesões em qualquer região do tegumento. Não acomete lesões pré existentes.

- Tipo de Lesão: eritematosa e infiltrada, sem ulceração e ausência de descamação.

- Comprometimento Neural: muito lento e, geralmente, assintomático.

- Tratamento: poliquimioterapia de forma irregular.

Esquematicamente, as diferenças entre as duas manifestações podem ser resumidas na Tabela I.

ERITEMA NODOSO HANSÊNICO (ENH) ou TIPO 2: envolve as formas HD, HDV e HV, reconhecidas como multibacilares, porque os pacientes apresentam grande número de bacilos. Foi, originalmente, descrito por Murata (Japão) ${ }^{(3)}$, em 1912, e, atualmente, é reconhecido como alteração da imunidade humoral, sendo exemplo de reação imunológica, com predomínio da reação por imuno complexo circulante (Tipo III), na classificação de Coombs \& Gell( ${ }^{(7)}$. ENH tende a ocorrer durante a poliquimioterapia, quando as lesões cutâneas estão em involução, entretanto, existem casos em que a reação tipo ENH é a primeira 
Tabela I: Diferenças entre RR e Recidiva

\begin{tabular}{|l|l|l|}
\hline & \multicolumn{1}{|c}{ Reação Reversa } & \multicolumn{1}{c}{ Recidiva } \\
\hline Início & Abrupto (poucas horas) & Lento (semanas, meses) \\
\hline Período & $\begin{array}{l}\text { Durante PQT, ou nos } 6 \text { meses pós-alta } \\
\text { terapêutica }\end{array}$ & $\begin{array}{l}\text { Geralmente, vários meses após alta } \\
\text { terapêutica }\end{array}$ \\
\hline Lesões cutâneas & $\begin{array}{l}\text { Atividade de lesões existentes com eritema } \\
\text { e edema }\end{array}$ & Não afetadas \\
\hline Lesões novas & Raras & Freqüientes \\
\hline Ulceração & Às vezes & Não \\
\hline Descamação & Freqüente & Ausente \\
\hline Comprometimento de nervo & Comum. Nervos espessados, dolorosos, & $\begin{array}{l}\text { Nervos podem ser envolvidos muito } \\
\text { lentamente }\end{array}$ \\
\hline Com alteração aguda da sensibilidade & Não comprometidas \\
\hline Resposta à corticoterapia & Raramente com febre e mal-estar geral & Sem alteração \\
\hline Adesão à poliquimioterapia & Boa, geralmente & Irregular \\
\hline
\end{tabular}

manifestação ${ }^{(5,12)}$, precedendo o diagnóstico de hanseníase, ou ainda ocorre após o tratamento específico. Não é muito raro encontrarmos pacientes com ENH durante ou após uso de antibióticos para tratamento de amigdalite, por exemplo, sugerindo que esse antibiótico teve alguma atividade sobre os bacilos, em indivíduo sem manifestação clínica de hanseníase, seguindo mecanismo semelhante ao observado durante o tratamento específico com a poliquimioterapia$\mathrm{PQT}^{(3)}$. Nesse tratamento, há destruição bacilar acentuada, liberação de frações antigênicas de M.leprae e indução da formação do anticorpo específico, anticorpo anti-glicolipídeo fenólico-1(anti PGL1) ${ }^{(13,14)}$, resultando na síndrome do imunocomplexo circulante, com depósito do complexo antígeno-anticorpo nos espaços teciduais e em vasos sangüíneos e linfáticos. Essas alterações imunológicas culminam com o aparecimento abrupto de nódulos e/ou placas infiltradas, dolorosas, de coloração rósea, que podem evoluir para pústulas e bolhas, com posterior ulceração e formação de necrose, nas formas mais graves do ENH, como no "eritema nodoso necrotizante"(3). As lesões préexistentes tendem a permanecer inalteradas. Há comprometimento do estado geral, o paciente apresenta febre e estado de toxemia, que pode vir acompanhado de infartamento ganglionar generalizado, dores musculares e ósseas (geralmente, dor tibial), artrite, irite, iridociclite, orquite, epididimite e neuropatia. Freqüentemente, encontra-se, no paciente com ENH, edema de mãos, pés e face. A reação inflamatória, neural tem características menos agressivas, devido à evolução mais lenta que a observada na reação reversa. Os exames laboratoriais podem mostrar:

- hematológico: leucocitose com neutrofilia e aumento do número de plaquetas ${ }^{(15,16)}$;

- aumento de proteínas da reação inflamatória aguda: proteína-C-reativa e a1 tripsina ${ }^{(17)}$;

- gamaglobulinas: aumento de $\operatorname{IgG}$ e $\operatorname{IgM}^{(3)}$;

- complemento: aumento das frações $\mathrm{C} 2$ e $\mathrm{C} 3^{(3)}$; proteinúria $^{(3)}$;

- histopatológico de lesão cutânea: infiltrado inflamatório, neutrofílico, perivascular, em derme reticular, compatível com vasculite; numerosos bacilos fragmentados e granulosos.

As reações de ENH podem regredir rapidamente, mas podem, também, persistir durante anos, como formas crônicas e recorrentes de ENH, que podem ter manifestações clínicas INTERMITENTES ou CONTÍNUAS $^{(3)}$, dependendo da capacidade de resposta inflamatória do paciente ${ }^{(18)}$. Reações intermitentes são aquelas que evoluem com duração de 1-2 semanas e permanecem sem reações pelo período de 1-2 meses. Podem ainda ser moderadas ou graves. 
ENH intermitente moderado é, geralmente, acompanhado de febre e comprometimento geral, moderado, com sintomas de neurite, especialmente espessamento e dor no nervo e em seu trajeto, porém sem perda da função. Enquanto, na forma grave do ENH intermitente, predomina febre elevada, com grave comprometimento geral, lesões pustulosas e/ou ulceradas, neurite acentuada e perda da função motora, edema em articulações e, em alguns casos, sinais de iridociclite ou orquite ${ }^{(3)}$.

Nas formas de ENH contínuo, não há período de remissão do quadro reacional, o paciente permanece durante toda a evolução com o quadro moderado ou grave, como descrito anteriormente, tornandose altamente debilitado e, se não conduzido adequadamente, pode ter evolução muito grave, que pode cursar com lesões hepáticas, renais, tromboembolia pulmonar e coagulação intravascular, disseminada ${ }^{(3,16)}$.

Muito raramente, as reações de ENH podem ser confundidas com o eritema papulo-nodular, manifestação de recidiva, nas formas multibacilares da hanseníase, que cursa com agravamento das lesões pré-existentes, aparecimento de novas lesões com baciloscopia positiva e evolução lenta. Quando surgem nódulos e pápulas, esse quadro não tem as características inflamatórias agudas do $\mathrm{ENH}$.

O diagnóstico diferencial do ENH deve ser feito, com outras causas de eritema nodoso, como infecções bacterianas, virais e fúngicas, farmacodermias, sarcoidose e linfomas.

Esquematicamente, as reações hansênicas podem ser diferenciadas segundo os parâmetros apresentados na Tabela II.

Quando houver lesão neural, sem inflamação da pele, deve ser prescrito o tratamento para reação reversa. Como regra geral, as lesões de ENH devem ser observadas para o diagnóstico de reação tipo 2 .

\section{TRATAMENTO DAS REAÇÕES HANSÊNICAS}

Deve-se fazer o diagnóstico clínico, especialmente de comprometimento neural, o mais precocemente possível e introduzir antiinflamatórios, especialmente os corticosteróides, imediatamente, para o sucesso do tratamento ${ }^{(2,3)}$. O interrogatório do paciente necessita ser dirigido, verificando-se se já tem diagnóstico de hanseníase e há quanto tempo, qual dose de PQT está tomando, se está sendo usado o esquema multi ou paucibacilar, se o surto reacional atual é o primeiro e se esse surto está relacionado a alguma doença, como diabetes, hepatopatia, nefropatia, stress físico e/ou psicológico, gestação, ou uso de algum medicamento.

Após o conhecimento de todos esses fatores, torna-se prioritário afastar as possíveis causas do quadro reacional e, em princípio, a manutenção da poliquimioterapia, segundo o esquema programado para o paciente. São pontos fundamentais para o êxito do tratamento do episódio reacional, além da introdução de antiinflamatórios não hormonais (AINH) ${ }^{(3)}$, a associação de medidas gerais, adjuvantes ao tratamento quimioterápico, como o repouso físico e mental, a hidratação, a imobilização, em posição de repouso, da região do nervo afetado, o uso de analgésico e, se necessária, dependendo da gravidade do quadro, a sedação ${ }^{(3)}$.

\section{REAÇÃO REVERSA OU REAÇÃO TIPO 1}

O objetivo do tratamento é reduzir a reação inflamatória, especialmente a neural. A neurite é

Tabela II: Diferenças entre Reação Reversa e Eritema Nodoso Hansênico

\begin{tabular}{|l|l|l|}
\hline Sinais & Reação Reversa (Tipo 1) & ENH (Tipo 2) \\
\hline Inflamação da pele & $\begin{array}{l}\text { Inflamação das lesões, restante do tegumento } \\
\text { inalterado }\end{array}$ & $\begin{array}{l}\text { Nódulos eritemato infiltrados, não } \\
\text { associados a lesões prévias. }\end{array}$ \\
\hline Condições gerais do paciente & Boas, sem febre & Febre alta, mal-estar geral \\
\hline Início e formas da doença & $\begin{array}{l}\text { Geralmente precoce, durante o tratamento. } \\
\text { Formas HDT , HD, HDV e HV. }\end{array}$ & $\begin{array}{l}\text { Geralmente com tratamento avançado. } \\
\text { Formas dimorfas(HD, HDV e HV). }\end{array}$ \\
\hline Olhos & Fraqueza de pálpebras. Dificuldade para fechar. & Irite e iridociclite.
\end{tabular}


associada à perda da sensibilidade e/ou à paralisia muscular.

1. Manter a poliquimioterapia, segundo esquema proposto $^{(2,3,5)}$.

2. Iniciar corticoterapia, sendo a prednisona a mais utilizada, na dose de 1 a $2 \mathrm{mg} / \mathrm{kg}$ de peso/ $\mathrm{dia}^{(2,3,5)}$, em dose única pela manhã, até que diminuam os sintomas de dor e a atividade reacional. A redução da dose de prednisona deve ser lenta, $5-10 \mathrm{mg} / 10$ 15 dias, até a dose de $5 \mathrm{mg} /$ dia, que deve ser mantida durante 10-15 dias antes da suspensão do medicamento. Os quadros reacionais, sem lesão neural, podem ser controlados com doses menores que 1,0 $\mathrm{mg} / \mathrm{kg} / \mathrm{dia}$ de prednisona ${ }^{(3)}$. A lesão clássica, acompanhada de neurite, deve ser tratada por períodos longos, de 4 a 9 meses de corticoterapia, como medi-da eficiente para prevenção de reações crônicas $^{(3)}$.

3. Imobilização da região afetada em posição de repouso. Caso a mão esteja afetada, deverá ser imobilizada, levemente inclinada, com o polegar em abdução. As neurites devem ser avaliadas por neurologistas, após a fase aguda, para indicação da conduta adequada, com o objetivo de serem evitadas as seqüelas ${ }^{(3)}$.

A indicação de antiinflamatório é feita porque o corticosteróide reduz o edema intraneural e, conseqüentemente, a compressão das fibras nervosas. $\mathrm{Pa}$ ralelamente, na RR, há acentuação da pressão sobre as fibras nervosas e a manutenção do tratamento da hanseníase será um fator adjuvante para a absorção da reação granulomatosa, levando à redução do processo inflamatório.

4. A cirurgia (neurolise) está indicada nos casos de abscesso de nervo e/ou dor neural, que não respondem ao tratamento com corticóide (prednisona), sendo, ainda, controversa sua indicação, como adjuvante de corticoterapia.

Atualmente, existem propostas de tratamento com drogas imunossupressoras, como azatioprina, na dose de $1 \mathrm{mg} / \mathrm{kg} / \mathrm{dia}$, ciclosporina e pulso de corticosteróide, porém não existem resultados definitivos e esses tratamentos encontram-se em fase de investigação $^{(3)}$.

\section{ERITEMA NODOSO HANSÊNICO (ENH) OU REAÇÃO TIPO 2}

O tratamento tem por objetivo reduzir a reação inflamatória generalizada, especialmente o quadro de toxemia e dor. Assim, nos casos em que a neurite não é o fator preponderante, as recomendações são as que vêm a seguir.

1. Talidomida ${ }^{(2,19)}$, na dose de $200-400 \mathrm{mg} /$ dia, em dose única diária, à noite, até redução dos sintomas. A indicação da talidomida baseia-se na ação da droga como inibidora de um dos fatores desencadeantes da reação inflamatória (citocina $\mathrm{TNFa})^{(20)}$, resultando no controle das alterações imunológicas e conseqüente melhora clínica.

2. Manter o tratamento específico com a poliquimioterapia, sem alteração do esquema proposto, com exceção dos casos com reações adversas a alguma das drogas ${ }^{(2)}$.

3. Nos casos de ENH persistente, pode se associar a clofazimina, $300 \mathrm{mg} / \mathrm{dia}$, pelo período de até $3 \mathrm{me}-$ $\operatorname{ses}^{(2,3,5,21)}$, devido ao seu efeito antiinflamatório e, também, pela ação bacteriostática sobre M.leprae, exercendo atividade terapêutica especial. A retirada da clofazemina deve ser feita lentamente, em período de até 3 meses. Deve ser lembrado que a ação da clofazimina é evidenciada, se prescrita junto à prednisona, porém, após a corticoterapia, seu efeito é discreto ${ }^{(3,5)}$.

4. Na presença de neurites acentuadas, deve ser introduzida a prednisona até a dose de $1 \mathrm{mg} / \mathrm{kg} / \mathrm{dia}$, seguindo-se as orientações descritas para a neurite da reação reversa.

5. Iridociclite aguda constitui emergência médica e deve ser seguida por especialista. $O$ tratamento com prednisona, como descrito, é uma das medidas terapêuticas ${ }^{(2)}$

6. Orquite e epididimite devem ser conduzidas com corticoterapia (prednisona $0,5-1,0 \mathrm{mg} / \mathrm{kg}$ dia), analgésico e repouso ${ }^{(2,3)}$.

7. Eritema nodoso necrotisante tem, como primeira escolha de tratamento, o corticóide, prednisona, $1,0 \mathrm{mg} / \mathrm{kg} / \mathrm{dia}^{(3,4)}$ associada a cuidados gerais para dor e neurites, especialmente. Em casos com predomínio de lesões de vasculite, pode ser associada a pentoxifilina, na dose de $400 \mathrm{mg}$ a cada $8 \mathrm{~h}^{(22)}$.

8. Em pacientes do sexo feminino, em idade fértil, é contra-indicado o uso da talidomida, devido aos seus efeitos teratogênicos ${ }^{(2)}$. A droga de primeira escolha é a prednisona, no esquema de 0,5 a $1,0 \mathrm{mg} / \mathrm{kg} /$ dia, em dose única pela manhã, até redução dos sintomas, seguida da retirada lenta. Nesses casos, a associação de clofazimina, conforme já descrito, é sempre interessante ${ }^{(3)}$ 
9. Quando a paciente é do sexo feminino, estando a talidomida contra-indicada ${ }^{(2)}$ a corticoterapia pode apresentar os efeitos colaterais, como diabetes, osteoporose, Cushing, e infecções oportunistas. Em tais situações, tenta- se associar a clofazimina, não ocorrendo controle do quadro de $\mathrm{ENH}$, pode ser prescrito o tratamento com azatioprina $1,0 \mathrm{mg} / \mathrm{kg} /$ dia ${ }^{(3)}$, iniciando-se a redução da prednisona após o controle da reação. Embora ainda em fase de estudos quanto à eficácia, tal esquema justifica-se pela necessidade de controle da reação, na tentativa de ser evitado o desenvolvimento de incapacidades.

10. Existem, atualmente, outros estudos à procura de novas opções de tratamento com imunossupressores, como ciclosporina, ciclofosfamida e pulsos de corticosteróides $^{(3,5)}$.

FOSS NT. Reactional episodes in leprosy. Medicina, Ribeirão Preto, 36: 453-459, apr./dec. 2003.

ABSTRACT - Reactional episodes are acute inflammatory reactions that suddenly appear on the chronic evolution of the leprosy disease. Currently these reactions represent a located or systemic exacerbation of the inflammatory reaction that can reach peripheral nerves, inducing a very painful situation which must be conducted with special care. The leprosy reactions are classified as Type1 Reaction or Reversal Reaction (RR) and Type2 Reaction or Erythema Nodosum Leprosum (ENL). The clinical and laboratories characteristics of these reactions, the diagnostic criteria and the main differences between the RR and ENL were presented, followed by the presentation of the approval conducts for the management of clinical alterations and the therapeutics ons for the two types of leprosy reactions.

UNITERMS - Leprosy. Reactional Episodes. Reversal Reactions. Erythema Nodosum Leprosum.

\section{REFERÊNCIAS BIBLIOGRÁFICAS}

1 - RIDLEY DS \& JOPLING WH. Classification of leprosy according to immunity. A five group system. Int J Lepr 34:255273, 1966.

2 - BRASIL. Ministério da Saúde. Guia para o Controle da Hanseníase - Cadernos de Atenção Básica $\mathbf{n}^{\circ}$ 10. Brasília, DF, 2002 (Série A. Normas e Manuais Técnicos; n¹11).

3 - YAWALKAR SJ. Reactions in Leprosy. In: YAWALKAR SJ. Leprosy for medical practioners and paramedical workers. World Health Organization - Novartis Foundation for Sustainable Development, Basle, Switzerland, Chapter 12: 72-79, 2002.

4 - VENKATESANK. Clinical pharmacokinetic considerations in the treatment of patients with leprosy. Clin Pharmacokinet 16:365-386, 1989.

5 - THE ILEP ACTION GROUP. How to recognise and manege leprosy reactions. The International Federation of Anti-Leprosy Associations, London, 2002.

6 - BECK-BLEUMINK M \& BERHE D. Occurrence of reactions, their diagnosis and management in leprosy patients treated with multidrug therapy. Experience in the leprosy control programme of the All Africa Leprosy and Reabilation Training Center (ALERT) in Ethiopia. Int J Lepr 60:173-184, 1992.
7 - COOMBS RRA \& GELL PGH. The classification of allergic reactions underlying disease in clinical aspects of immunology. Davis, Philadelphia, 1963.

8 - GEBRE S; SAUNDERSON P \& BYASS P. Relapses after fixed duration of multiple drug therapy: The AMFES Cohort. Lepr Rev 71: 85-98, 2000.

9 - GIRDHAR BK; GIRDHAR A \& KUMAR A. Relapses in multibacillary leprosy patients. Lepr Rev 71:144-153, 2000.

10 - CUNHA MGS. Episódios reacionais e relação com recidiva em doentes com hanseníase tratados com diferentes esquemas terapêuticos. Tese de Doutorado, Faculdade de Medicina de Ribeirão Preto da USP, Ribeirão Preto, 2001.

11 - GALLO MEN \& OLIVEIRA MLW. Recidivas e reinfecção em hanseníase. Medicina, Ribeirão Preto 30: 351-357, 1997.

12 - SAUNDERSON P; GEBRE S \& BYASS P. ENL reactions in the multibacillary cases of the AMFES Cohort in central Ethiopia: incidence and risk factors. Lepr Rev 71: 101-115, 2000.

13 - FOSS NT; CALLERA F \& ALBERTO FL. Anti PGL1 levels in leprosy patients and their contacts Braz $\mathbf{J}$ Med Biol Res 26:43-51, 1993.

14 - FOSS NT. Aspectos imunológicos da hanseníase. Medicina, Ribeirão Preto, 30: 335-339, 1997. 
15 - REA TH. Decreases in mean hemoglobin and serum albumin values in erythema nodosum leprosum. Int $\mathbf{J}$ Lepr Other Mycobact Dis 69:318-324, 2001.

16 - REA TH. Elevatede platelet counts and trombocytosis in erythema nodosum leprosum. Int $\mathbf{J}$ Lepr Other Mycobact Dis 70:167-173, 2002.

17 - FOSS NT; OLIVEIRA EB \& SILVA CL. Correlation between TNFa production, increase of plasma C-reactive protein level and suppression of $\mathrm{T}$ lymphocyte response to concanavalin A during erythema nodosum leprosum. Int $\mathbf{J}$ Lepr Other Mycobact Dis 61:218-225, 1993.

18 - TRAO VT; LONG HT; QUYEN ND; LY HM; HONG ND; HANG LT; KHANG DD; DUE LK; HENDRIKS JT \& WRIGERT P. Long term evaluation of the immune status in leprosy patients undergoing multiple drug therapy. Int J Lepr 62: 365-373, 1994.
19 - TEO SK, RESZTAK KE, SCHEFFLER MA, KOOK KA, ZELDIS JB, STIRLING DI \& THOMAS SD. Thalidomide in the treatment of leprosy. Microbes Infect 4:1193-1202, 2002.

20 - SAMPAIO EP; HERNANDEZ MO; CARVALHO DS \& SARNO EN. Management of erythema nodosum leprosum by thalidomide: thalidomide analogues inhibit M.leprae-induced TNFa production in vitro. Biomed Pharmacother 56:13-19, 2002.

21 - GALLO MEN. Poliquimioterapia com duração fixa em hanseníase multibacilar. Tese de Doutorado, Fundação Oswaldo Cruz. Instituto Oswaldo Cruz, Rio de Janeiro, 1998.

22 - DE CARSALADE GY; ACHIRAFI A \& FLAGEUL B. Pentoxifyline in the treatment of erythema nodosum leprosum. J Dermatol 30: 64-68, 2003. 